\title{
A case of intra-articular angioleiomyoma of the talocrural joint
}

\author{
IRENE THUNG ${ }^{1}$, SEPI MAHOOTI², XIANGDONG XU² \\ ${ }^{1}$ Division of Anatomic Pathology, Department of Pathology, University of California, San Diego Medical Center, San \\ Diego, CA, USA \\ ${ }^{2}$ Division of Anatomic Pathology, Department of Pathology, VA San Diego Healthcare System, San Diego, CA, USA
}

\begin{abstract}
Angioleiomyoma is a benign pericytic neoplasm with smooth muscle differentiation. Intra-articular angioleiomyoma is exceptionally rare with only four cases reported, all involving the knee joint. Here we report the first case of intra-articular angioleiomyoma entirely localized within the ankle joint. An 83-year-old male presented with progressively worsening ankle pain. Subsequent magnetic resonance imaging showed a soft tissue mass within the talocrural joint. Histologic examination and ancillary testing demonstrated fascicles of smooth muscle cells and interspersed, often compressed, vascular channels, consistent with an angioleiomyoma. This case highlights the importance of including angioleiomyoma in the differential diagnosis of an ankle joint mass.
\end{abstract}

Keywords: intra-articular, ankle, angioleiomyoma, pericytic neoplasm, soft tissue mass.

\section{Introduction}

Pericytic tumors differentiate toward perivascular contractile cells and include angioleiomyomas, glomus tumors, and myopericytomas.

Angioleiomyomas are usually located in the dermis of the lower extremities, but can occur anywhere in the body (1). While angioleiomyomas are a fairly common type of neoplasm, intra-articular localization is rare, with only four reported cases, all of which occurred in

\footnotetext{
Corresponding Author:

Xiangdong $\mathrm{Xu}, \mathrm{MD}, \mathrm{PhD}$

3350 La Jolla Village Drive, Mail-113,

San Diego, CA 92161, USA

E-mail: Xiangdong.Xu@va.gov
}

the knee joint (2-5). Here we describe the first case of an intra-articular angioleiomyoma of the anterior ankle joint.

\section{Case presentation}

An 83-year-old male presented with a two-year history of right ankle pain. The pain progressively worsened over a six-month period, with the development of a large, unexplained effusion. Analysis of the joint aspirate was negative for crystals or infection.

Although radiographic imaging was negative for any bony abnormalities, magnetic resonance imaging (MRI) revealed a $4.8 \times 2.6 \times 1.4 \mathrm{~cm}$ intra-articular mass within the anterior talocrural joint causing anteromedial osseous erosion. The soft tissue mass was isointense to mildly hyperintense to muscle on T1-weighted sequences and slightly hyperintense on T2-weighted sequences (Fig. 1). The mass showed contrast enhancement with gadolinium. Radiologically, pigmented villonodular synovitis was considered in the differential diagnosis.
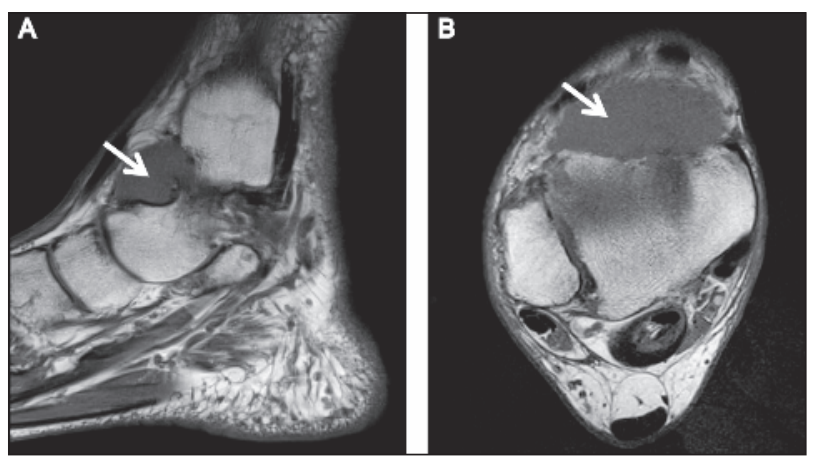

Figure 1. Magnetic resonance imaging of the right ankle without contrast. T1-weighted sagittal (A) and axial (B) images reveal an intra-articular mass (arrow) in the ankle that is mildly hyperintense to muscle. 
Intraoperatively, we observed an entirely intra-articular, well-circumscribed, firm tumor, located deep to the extensor retinaculum. Although superficial erosions of the tibia and talus were visible, the tumor did not grow into the bone.

Grossly, the specimen was an encapsulated firm nodular mass that measured $4.5 \times 2.5 \times 2.2 \mathrm{~cm}$. Serial sectioning demonstrated white to tan homogenous cut surfaces. Microscopically, the mass contained fascicles of smooth muscle associated with compressed vascular channels (Fig. 2 A). Examination at higher magnification showed that the spindle cells had bland cigar- shaped nuclei with blunt ends (Fig. 2 B). Areas with hyalinized changes were identified. However, no significant cytological atypia, mitotic figures or necrosis were noted.

Immunohistochemistry showed spindle cells strongly positive for desmin, smooth muscle actin, and smooth muscle myosin heavy chain, confirming smooth muscle differentiation (Fig. 2 C-D).

These cells were negative for S 100 with Ki-67 immunostain demonstrated a low proliferation rate of less than $1 \%$. Overall, the findings are consistent with an intra-articular angioleiomyoma, solid subtype.
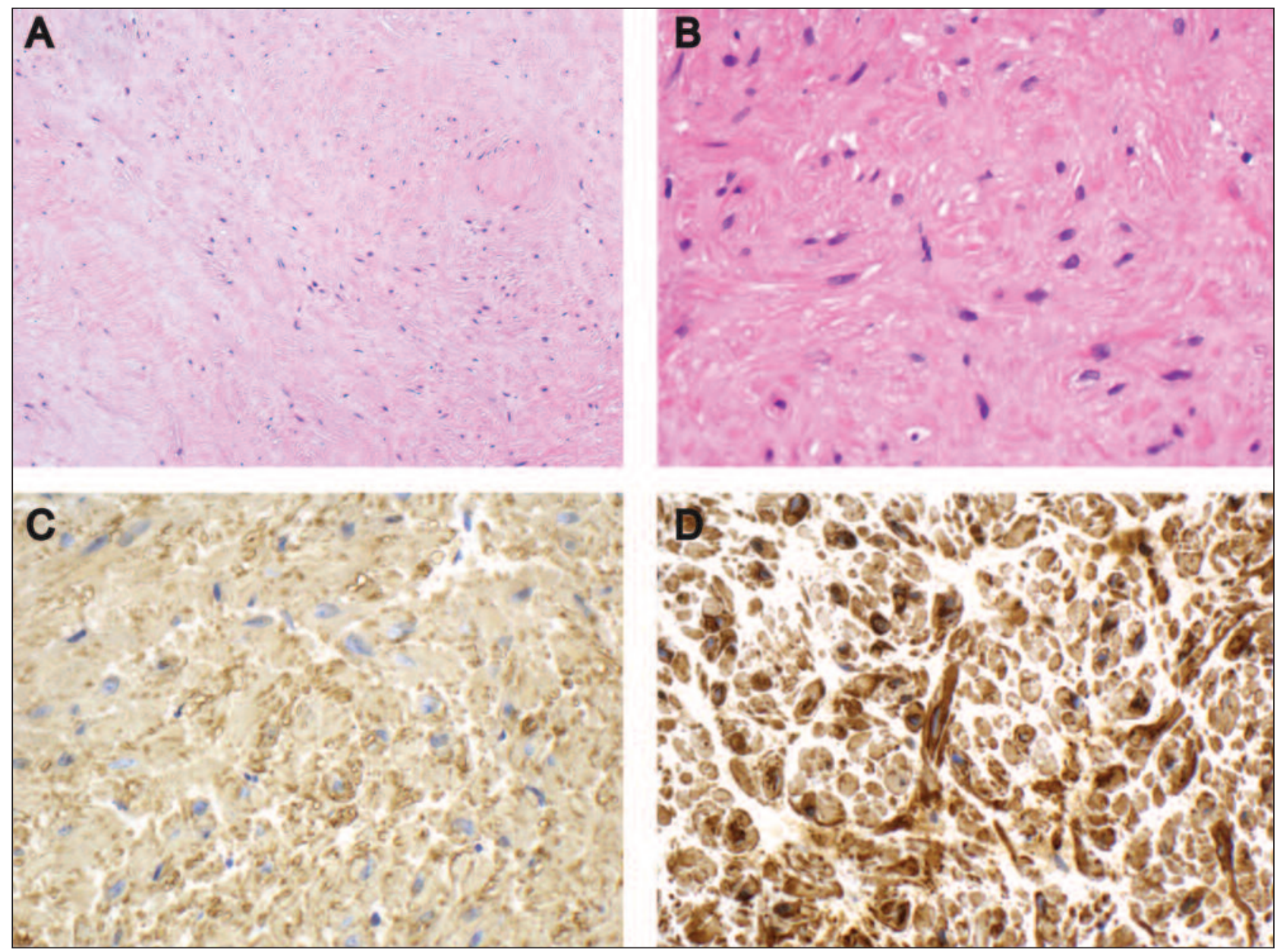

Figure 2. Histologic and immunohistochemical features of the angioleiomyoma. Hematoxylin \& eosin stained slides reveal a spindle cell proliferation with compressed vascular channels and bland spindle cells (A, original magnification 100x) with short-spindly, cigar-shaped nuclei (B, original magnification 400x). Immunohistochemistry demonstrates that the spindle cells are positive for smooth muscle actin (C, original magnification $400 \mathrm{x}$ ), and smooth muscle myosin (D, original magnification $400 \mathrm{x}$ ). 


\section{Discussion}

Soft tissue masses of the ankle are rare with a wide differential diagnosis including tumor-like lesions and neoplasms. The most common tumor-like lesion types occurring in the ankle include ganglion cyst, epidermoid cyst, and pigmented villonodular synovitis. Neoplasms of the ankle joint include hemangioma, lipoma, neural fibrolipoma, deep fibromatosis, and leiomyosarcoma (6-8). Angioleiomyoma is a subcutaneous or deep dermal neoplasm with a predilection for the extremities. Clinically, this entity presents as painful, slow-growing firm mass frequently sensitive to temperature. There are three different histological subtypes: solid, venous, and cavernous. In our case, the mass was composed of bundles of smooth muscle cells associated with mostly compressed vascular channels, and was most consistent with the solid subtype. Intra-articular angioleiomyomas are an exceedingly rare occurrence. To date, only four cases of intra-joint angioleiomyoma have been reported in the English literature, all of which involved the knee joint $(2-5)$. Our case is the first angioleiomyoma within the ankle joint. The intra-joint location was confirmed by both MRI imaging and intraoperative observation. Surgical resection of angioleiomyomas is curative and local recurrence is rare (1).
In conclusion, angioleiomyoma can involve the ankle joint. Therefore, it needs to be considered in the differential diagnosis of an ankle joint mass.

\section{References}

1. Hisaoka M, Quade B. World Health Organization Classification of Tumours: Pathology and Genetics of Tumours of Soft Tissue and Bone. Lyon, IARC Press. 2013;120-121.

2. Fukawa T, Akatsu Y, Sasho T. Intra-articular angioleiomyoma of the knee with an atypical finding on gadolinium-enhanced magnetic resonance imaging: a case report. J Med Case Rep. 2014:8:238.

3. Al-Jabri T, Garg S, Rao S. A rare case of angioleiomyoma of the knee: a case report. Cases J. 2009;2:7885.

4. Okahashi K, Sugimoto K, Iwai M, et al. Intra-articular angioleiomyoma of the knee: a case report. Knee. 2006;13:330332.

5. Gulati M, Kapoor A, Maheshwari J. Angiomyoma of the knee joint: value of magnetic resonance imaging. Australas Radiol. 1999;43:353-354.

6. Llauger J, Palmer J, Monill JM, et al. MR imaging of benign soft-tissue masses of the foot and ankle. Radiographics. 1998;18:1481-1498.

7. Akisue T, Matsumoto K, Yamamoto T, et al. Neural fibrolipoma of the superficial peroneal nerve in the ankle: a case report with immunohistochemical analysis. Pathol Int. 2002;52:730-733.

8. Woertler K. Soft tissue masses in the foot and ankle: characteristics on MR imaging. Semin Musculoskelet Radiol. 2005;9:227-242. 